\title{
A Characterization of Gram Matrices of Polytopes
}

\author{
R. Díaz \\ Dpto. de Geometría y Topología, Facultad de CC. Matemáticas, \\ Universidad Complutense, 28040 Madrid, Spain \\ radiaz@eucmos.sim.ucm.es
}

\begin{abstract}
We give a characterization of the Gram matrices of spherical and finite-volume hyperbolic polytopes of a given combinatorial type. This is done in terms of the signs of certain minors of the Gram matrix.
\end{abstract}

\section{Introduction}

Coxeter [Co] and Lannér [La] gave a complete description of the possible dihedral angles of arbitrary dimensional simplices with angles not exceeding $\pi / 2$ in a space of constant curvature. This produces a description of all spherical and bounded euclidean polytopes with dihedral angles not exceeding $\pi / 2$, since these polytopes are direct products of simplices [Co]. Andreev [An1], [An2] established the analogous description for finite-volume hyperbolic polytopes of dimension three (i.e., polyhedra): for a given combinatorial type of polyhedron, not a simplex, he determined necessary and sufficient conditions on the dihedral angles under which there exists a finite volume hyperbolic polyhedron with angles not greater than $\pi / 2$ and with the given combinatorial type. All these conditions are just linear inequalities. A reason to describe polytopes from their dihedral angles is that bounded hyperbolic polytopes of dimension greater than two with dihedral angles not exceeding $\pi / 2$ are determined by their dihedral angles up to isometry [An1]. This is mainly deduced from Cauchy's lemmas [Ca] (see also [Al] and [RH] for proofs and other uniqueness results).

Any hyperbolic or spherical polytope can also be determined up to isometry by its Gram matrix (except one indeterminacy case, see Section 1). Each dihedral angle of the polytope is in correspondence with a specific entry of this matrix, namely, this entry is equal to minus the cosine of the dihedral angle. According to a result of Andreev [An3], all off-diagonal entries of the Gram matrix of a polytope with dihedral angles not greater than $\pi / 2$ are nonpositive. Using the properties of matrices with nonnegative 
entries (essentially the Perron-Fröbenius Theorem), Vinberg characterized the Gram matrices of polytopes of arbitrary dimension with dihedral angles not exceeding $\pi / 2$ [Vi1], [Vi3]. He also described the combinatorial type of the polytope corresponding to such a matrix in terms of the signs of principal minors of this matrix.

When there is no restriction on the values of the dihedral angles, the characterization of Gram matrices of simplices is easily established in terms of the signs of certain minors (see [Vi2] or [Mi]). For an arbitrary combinatorial type of polyhedron, Rivin characterized the dihedral angles of ideal hyperbolic polyhedra (i.e., with all their vertices on the sphere at infinity) of a given combinatorial type [Ri1], [Ri2]. As in Andreev's theorems, Rivin's conditions form a system of linear inequalities.

In this paper we give a characterization of the Gram matrices of spherical and finitevolume hyperbolic polytopes of a given combinatorial type, without any restriction on the dihedral angles. As in the case of simplices, this is done in terms of the signs of certain (not only principal) minors of the Gram matrix. Both characterizations are consequence of a general result (Theorem 4.1) for cones over polytopes (polyhedral cones) in a vector space with a quadratic form given in it. This directly produces the result for spherical polytopes. For the hyperbolic case, it is still necessary to study the relative position between the polyhedral cone and the hyperbolic space. Therefore, extra conditions are required in order to obtain a finite-volume polytope (Theorem 4.4). The classical result for simplices can be easily obtained as a corollary (Corollary 4.5). The techniques in the proof of these results are based on a combinatorial description of the orientation of a polytope by means of what we call maximal oriented cycles (see Section 2). These cycles will be used to determine the combinatorial type of the polytope (see Section 3).

The organization of this paper is as follows. In Section 1 we review some general facts on polyhedral cones. In Section 2 we study different aspects (topological, combinatorial, and vectorial) relative to orientations on polytopes and prove Proposition 2.4 relating these concepts. This result is used for proving the necessary condition of Theorem 4.1. Section 3 is devoted to the proof of Proposition 3.3, which gives sufficient conditions for two sets of vectors to determine the facets (i.e., maximal dimensional faces) and vertices of a polytope of a given combinatorial type; this is used to prove the sufficient condition of Theorem 4.1. In Section 4, we state and prove our main results. Finally, we comment on some applications in Section 5 and give an example to illustrate how to use our results to characterize the dihedral angles of a hyperbolic or spherical polytope.

\section{Polyhedral Cones}

We recall some basic definitions concerning polytopes and polyhedral cones. See [Gr], [BL], [Vi1], and [Vi3] for more details.

Definitions. A polytope (or affine polytope) is a bounded subset $P \subset \mathbf{R}^{d}$ defined as the intersection of finitely many closed halfspaces. The dimension of the polytope is the dimension of its affine span. A polytope of dimension $d$ is called a $d$-polytope. We use the term polyhedron to refer to a 3-polytope. A face is the intersection of the polytope with a hyperplane which bounds a halfspace containing the polytope. This includes the empty set. By convention, $P$ will also be a face. These two faces are called improper, 
and the remaining faces are called proper. Faces are also polytopes; vertices are faces of dimension zero, and proper faces of maximal dimension are called facets. If $F$ and $F^{\prime}$ are faces of $P$, we write $F \prec F^{\prime}$ to denote that $F$ is contained in $F^{\prime}$. The set of all faces of a polytope $P$ except $P$ itself, with this order relation, is a lattice, called the combinatorial type of $P$ and denoted by $\mathcal{P}$. We always consider polytopes with labeled faces, that is, we number the facets. Two polytopes have the same combinatorial type if there is a lattice isomorphism between the corresponding combinatorial types which preserves the numbering of the facets.

We use italic capital letters $P, F, C, V, \ldots$ for affine polytopes and their faces, and caligraphic letters $\mathcal{P}, \mathcal{F}, \mathcal{C}, \mathcal{V}, \ldots$ for the corresponding elements of the combinatorial type of the polytope.

The following result is readily verified $[\mathrm{Gr}]$ :

Lemma 1.1. Let $P$ be a polytope. Then

(C1) any face is contained in some facet;

(C2) any face of dimension $d-2$ is contained in exactly two different facets;

(C3) any two facets $F, F^{\prime}$ can be joined by a chain of adjacent facets (i.e., there exists a finite sequence $F=F_{1}, \ldots, F_{r}=F^{\prime}$ of facets such that, for all $i, F_{i} \cap F_{i+1}$ is a facet of both $F_{i}$ and $F_{i+1}$ ).

If we embed $\mathbf{R}^{d}$ as an affine subspace of the vector space $\mathbf{R}^{d+1}$ not containing the origin, then the (positive) cone over a polytope $P$ is called a polyhedral cone. More precisely we have the following:

Definitions. A polyhedral cone in the vector space $\mathbf{R}^{d+1}$ is a subset of the form

$$
\hat{P}=\bigcap_{i=1}^{n} \hat{H}_{i}^{-},
$$

where $\hat{H}_{i}^{-}$is a closed halfspace bounded by the linear hyperplane $\hat{H}_{i}$ and such that the intersection of the hyperplanes $\hat{H}_{i}$ is the origin of $\mathbf{R}^{d+1}$. It can be seen that this last nondegeneracy condition assures that there exists an affine hyperplane $A$ such that $P=\hat{P} \cap A$ is bounded, and therefore a polytope. We always assume that $\hat{P}$ has nonempty interior and that none of the halfspaces $\hat{H}_{i}^{-}$contains the intersection of the others. The faces of a polyhedral cone $\hat{P}$ are defined to be the cone over the faces of the polytope $P=\hat{P} \cap A$. The combinatorial type of $\hat{P}$ is defined to be that of $P$.

Let $f$ be a nondegenerate quadratic form (we also denote by $f$ the associated bilinear form). We say that $f(v)$ is the norm of the vector $v \in \mathbf{R}^{d+1}$. A subspace $\hat{H} \subset \mathbf{R}^{d+1}$ is called lightlike if the quadratic form $f$ restricted to it is degenerate. An orthogonal transformation is a linear isomorphism that preserves $f$. We call the pair $\left(\mathbf{R}^{d+1}, f\right)$ a geometric space.

If the hyperplane $\hat{H}_{i}$ is not lightlike, then the outward normal vector to the halfspace $\hat{H}_{i}^{-}$is the unique vector $e_{i}$ with $f\left(e_{i}\right)= \pm 1$ and verifying

$$
\hat{H}_{i}^{-}=\left\{x \in \mathbf{R}^{d+1} \mid f\left(x, e_{i}\right) \leq 0\right\} .
$$


Let $\hat{P}=\bigcap_{i=1}^{n} \hat{H}_{i}^{-}$be a polyhedral cone such that no $\hat{H}_{i}$ is lightlike. The Gram matrix of $\hat{P}$ is defined to be the matrix of inner products of the outward normal vectors to the halfspaces $\hat{H}_{i}^{-}$, that is,

$$
G(\hat{P})=\left(f\left(e_{i}, e_{j}\right)\right)_{i, j=1, \ldots, n} .
$$

The nondegeneracy condition on the polyhedral cone implies that rk $G(\hat{P})=d+1$, and so the Gram matrix determines the polyhedral cone up to orthogonal transformation.

We are mainly interested in two particular values of the signature of $f$, those defining either the spherical or hyperbolic geometry. If $f$ is the usual euclidean inner product, then the $d$-dimensional sphere $\mathbf{S}^{d}$ is the set $f^{-1}(1)$. A spherical polytope is the intersection of the sphere with a polyhedral cone.

Similarly, for hyperbolic geometry, we take $f$ to have signature $(d, 1)$. More precisely, let $\left(x_{1}, \ldots, x_{d}, x_{d+1}\right)$ be the coordinates of the vector $x$ with respect to the canonical basis of $\mathbf{R}^{d+1}$ and let $f$ be the quadratic form defined by $f(x)=x_{1}^{2}+\cdots+x_{d}^{2}-x_{d+1}^{2}$. Then the hyperbolic space is the upper sheet of the hyperboloid $f^{-1}(-1)$ :

$$
\mathbf{H}^{d}=\left\{x \in \mathbf{R}^{d+1} \mid f(x)=-1, x_{d+1}>0\right\} .
$$

The sphere at infinity, denoted $\partial \mathbf{H}^{d}$, is the set of rays of the set $\left\{x \in \mathbf{R}^{d+1} \mid f(x)=\right.$ $\left.0, x_{d+1} \geq 0\right\}$. A hyperbolic isometry is an orthogonal transformation that preserves $\mathbf{H}^{d}$. A hyperbolic polytope is the intersection of a polyhedral cone $\hat{P}=\bigcap_{i=1}^{n} \hat{H}_{i}^{-}$with $\mathbf{H}^{d}$, i.e.,

$$
P=\left(\bigcap_{i=1}^{n} \hat{H}_{i}^{-}\right) \cap \mathbf{H}^{d}=\bigcap_{i=1}^{n} H_{i}^{-},
$$

where all the facets of $\hat{P}, \hat{H}_{i} \cap \hat{P}$, have nonempty intersection with $\mathbf{H}^{d}$ (we have used the notation $H_{i}^{-}=\hat{H}_{i}^{-} \cap \mathbf{H}^{d}$ ).

A hyperbolic polytope $P=\hat{P} \cap \mathbf{H}^{d}$ has finite volume if and only if the polyhedral cone $\hat{P}$ is contained in the set $\left\{x \in \mathbf{R}^{d+1} \mid f(x) \leq 0, x_{d+1} \geq 0\right\}$. The combinatorial type and the Gram matrix of a spherical or hyperbolic polytope are the combinatorial type and the Gram matrix of the polyhedral cone mentioned above. We note that all the diagonal entries of this matrix are equal to one. Finite-volume hyperbolic polytopes are determined up to isometry by their Gram matrices.

In the remainder of this section we discuss the method we use to determine the vertices of a given polyhedral cone. Recall that if $\hat{P}=\bigcap_{i=1}^{n} \hat{H}_{i}^{-} \subset\left(\mathbf{R}^{d+1}, f\right)$ is a polyhedral cone over an affine polytope $P$, then a vertex of $\hat{P}$ is the ray $\hat{v}$ spanned by a vertex $v$ of $P$. By abuse of notation, any nonzero vector in this ray is also called vertex.

It is convenient later to choose a single vector (in fact, one of the two rays) in the line $L(v)=L\left(e_{i_{1}}, \ldots, e_{i_{d}}\right)^{\perp}$, in the same way as the vector product in $\mathbf{R}^{3}$ determines a single vector orthogonal to two given vectors. We do it by using the Hodge star operator. We recall its definition and some properties (see, for instance, [Iv]).

First recall the quadratic form $\bigwedge^{m} f$ defined in the space $\bigwedge^{m} \mathbf{R}^{d+1}$ as follows:

$$
\left(\bigwedge^{m} f\right)\left(e_{1} \wedge \cdots \wedge e_{m}, v_{1} \wedge \cdots \wedge v_{m}\right)=\operatorname{det}\left(f\left(e_{i}, v_{j}\right)\right)_{i, j=1, \ldots, m}
$$


We fix an orientation in $\mathbf{R}^{d+1}$ and call it positive. For each $m$, the Hodge star operator is an isomorphism $\star$ between the spaces $\bigwedge^{m} W$ and $\bigwedge^{d+1-m} W$. For $m=d+1$, this isomorphism is determined by the condition that, for any positively oriented orthonormal basis $u_{1}, \ldots, u_{d+1}$ of $\mathbf{R}^{d+1}$, it holds that $\star\left(u_{1} \wedge \cdots \wedge u_{d+1}\right)=1$. We observe that $\star\left(e_{1} \wedge \cdots \wedge e_{d+1}\right)$ is equal to the determinant of the matrix of coordinates of the vectors $e_{i}$ with respect to any positively oriented orthonormal basis of $\mathbf{R}^{d+1}$.

For $m \leq d$, the Hodge star operator $\star$ is defined by the composition

$$
\bigwedge^{m} W \stackrel{\psi}{\longrightarrow}\left(\bigwedge^{d+1-m} W\right)^{\vee} \stackrel{\tau^{-1}}{\longrightarrow} \bigwedge^{d+1-m} W,
$$

where $V^{\vee}$ denotes the dual space of the vector space $V$ and $\psi$ and $\tau$ are the isomorphisms defined by

$$
\begin{aligned}
& \psi(e)(w)=\star(e \wedge w) \quad \text { for } \quad e \in \bigwedge^{m} W, \quad w \in \bigwedge^{d+1-m} W \\
& \tau(v)(w)=\left(\bigwedge^{d+1-m} f\right)(v, w), \quad \text { for } \quad v, w \in \bigwedge^{d+1-m} W
\end{aligned}
$$

(i.e., $\tau$ is the polarity for the quadratic form $\left.\bigwedge^{d+1-m} f\right)$. The vector $\star\left(e_{1} \wedge \cdots \wedge e_{d}\right)$ is orthogonal to each $e_{i}$ and its norm is equal to the norm of $e_{1} \wedge \cdots \wedge e_{d}$, up to the sign. Also, the orientation of the basis $e_{1}, \ldots, e_{d}, \star\left(e_{1} \wedge \cdots \wedge e_{d}\right)\left(\right.$ whenever $\left.\wedge^{d} f\left(e_{1} \wedge \cdots \wedge e_{d}\right) \neq 0\right)$ depends on det $f$ and the sign of $\wedge^{d} f\left(e_{1} \wedge \cdots \wedge e_{d}\right)$, where we have denoted by det $f$ the determinant of the matrix of $f$ with respect to any orthonormal basis. This is precisely stated in the following lemma.

Lemma 1.2. Let $e_{1}, \ldots, e_{d}, v_{1}, \ldots, v_{d} \in \mathbf{R}^{d+1}$; then:

(a) $f\left(\star\left(e_{1} \wedge \cdots \wedge e_{d}\right), v\right)=\star\left(e_{1} \wedge \cdots \wedge e_{d} \wedge v\right)$,

(b) $\left(\wedge^{d} f\right)\left(v_{1} \wedge \cdots \wedge v_{d}, e_{1} \wedge \cdots \wedge e_{d}\right)=(\operatorname{det} f) \star\left(v_{1} \wedge \cdots \wedge v_{d} \wedge \star\left(e_{1} \wedge \cdots \wedge e_{d}\right)\right)$,

(c) $\left(\wedge^{d} f\right)\left(e_{1} \wedge \cdots \wedge e_{d}, v_{1} \wedge \cdots \wedge v_{d}\right)=(\operatorname{det} f) f\left(\star\left(e_{1} \wedge \cdots \wedge e_{d}\right), \star\left(v_{1} \wedge \cdots \wedge v_{d}\right)\right)$.

\section{Orientation}

We recall [BL] that a complete flag $\mathcal{B}$ on the combinatorial type $\mathcal{P}$ of a $d$-polytope is a family $\emptyset=\mathcal{F}_{-1} \prec \mathcal{F}_{0} \prec \cdots \prec \mathcal{F}_{d-1}$ of faces such that there is a face of each dimension and the face $\mathcal{F}_{i}$ of dimension $i$ is contained in the face $\mathcal{F}_{i+1}$ of dimension $i+1$.

Let $\mathcal{B}: \emptyset=\mathcal{F}_{-1} \prec \mathcal{F}_{0} \prec \cdots \prec \mathcal{F}_{d-1}$ be a complete flag. For all $i=0, \ldots, d-1$ we take a vertex $\mathcal{V}_{i} \in \mathcal{F}_{i} \backslash \mathcal{F}_{i-1}$ and consider a vertex $\mathcal{V}_{d} \notin \mathcal{F}_{d-1}$. We call $\mathcal{V}_{0}, \ldots, \mathcal{V}_{d}$ a basis of $\mathcal{P}$ associated to the flag $\mathcal{B}$.

Definition. Two complete flags $\mathcal{B}: \emptyset \prec \mathcal{F}_{0} \prec \cdots \prec \mathcal{F}_{d-1}$ and $\mathcal{B}^{\prime}: \emptyset \prec \mathcal{F}_{0}^{\prime} \prec$ $\cdots \prec \mathcal{F}_{d-1}^{\prime}$ on the combinatorial type of a $d$-polytope differ by an elementary step if they only differ in a face, namely, there exists a label $i \in\{0, \ldots, d-1\}$ such that $\mathcal{F}_{i} \neq \mathcal{F}_{i}^{\prime}$ and $\mathcal{F}_{j}=\mathcal{F}_{j}^{\prime}$ for all $j \neq i$. A chain of complete flags of length $m$ is a finite sequence $\mathcal{B}_{0}, \mathcal{B}_{1}, \ldots, \mathcal{B}_{m}$ of complete flags such that any two consecutive flags differ by an elementary step. 
Remarks. If two complete flags $\mathcal{B}$ and $\mathcal{B}^{\prime}$ differ by an elementary step, then, by Lemma $1.1(\mathrm{C} 2), \mathcal{B}^{\prime}$ is completely determined by $\mathcal{B}$ and the dimension of the faces that are different. Using Lemma 1.1 (C3) and an easy induction argument on the dimension of the polytope, it is not difficult to check that given two complete flags there is always a chain joining them.

Definition. (a) Let $\mathcal{P}$ be the combinatorial type of a $d$-polytope. A maximal oriented cycle is a family $\mathcal{C}_{1}, \ldots, \mathcal{C}_{d}, \mathcal{C}_{d+1}$ of $d+1$ ordered facets of $\mathcal{P}$ which "meets a complete flag"; this means that setting $\mathcal{F}_{i}=\mathcal{C}_{1} \cap \cdots \cap \mathcal{C}_{d-i}$ for $i=-1, \ldots, d-1$, then $\mathcal{B}$ : $\emptyset=\mathcal{F}_{-1} \prec \mathcal{F}_{0} \prec \ldots \prec \mathcal{F}_{d-1}$ is a complete flag. We also say that $\mathcal{C}_{1}, \ldots, \mathcal{C}_{d}, \mathcal{C}_{d+1}$ is a maximal oriented cycle incident to the flag $\mathcal{B}$ or to the vertex $\mathcal{F}_{0}$. Equivalently, $\mathcal{C}_{1}, \ldots, \mathcal{C}_{d}, \mathcal{C}_{d+1}$ is a maximal oriented cycle incident to the flag $\mathcal{B}$ if $\mathcal{C}_{1}=\mathcal{F}_{d-1}$ and, for $i=2, \ldots, d+1$, the facet $\mathcal{C}_{i}$ contains $\mathcal{F}_{d-i}$ but does not contain $\mathcal{F}_{d-i+1}$.

(b) Let $\mathcal{C}_{1}, \ldots, \mathcal{C}_{d}, \mathcal{C}_{d+1}$ be a maximal oriented cycle incident to a flag $\mathcal{B}: \emptyset=\mathcal{F}_{-1} \prec$ $\mathcal{F}_{0} \prec \ldots \prec \mathcal{F}_{d-1}$. The family $\mathcal{C}_{1}, \ldots, \mathcal{C}_{d}$ is called an oriented cycle incident to $\mathcal{B}$ or to the vertex $\mathcal{F}_{0}$; for $r=1, \ldots, d-1$, the family $\mathcal{C}_{1}, \ldots, \mathcal{C}_{r}$ is called a truncated oriented cycle incident to the face $\mathcal{F}_{d-r}$. Note that we can consider oriented cycles and maximal oriented cycles as truncated cycles incident, respectively, to a vertex or the empty set.

We can also regard $\mathcal{P}$ as a linear convex cell complex in the sense of piecewise-linear topology (see, for instance, $[\mathrm{Hu}]$ and $[\mathrm{RS}]$ ). In this context, a cell is the same as an affine polytope, and a linear convex cell complex (or simply cell complex) $K$ is a finite set of cells in some $\mathbf{R}^{m}$ satisfying

(i) if $A \in K$ and $B \prec A$, then $B \in K$,

(ii) if $A, B \in K$, then $A \cap B$ is a face of both $A$ and $B$.

We refer to the faces of maximal dimension of a complex $K$ as its facets. We will see now how an oriented cycle induces a topological orientation on the underlying space $|\mathcal{P}|$ of $\mathcal{P}$, which is a sphere of dimension $d-1$, and so an orientable manifold. Given two embeddings of $h_{1}, h_{2}$ of $[-1,1]^{m}$ in the interior of a connected $m$-manifold $M$, then $h_{1}$ is ambient isotopic to either $h_{2}$ or $h_{2} \circ r$, where $r$ is a reflexion of $[-1,1]^{m}$ (Disk Theorem, see [RS]). Therefore, there are one or two equivalence classes of embeddings of $I^{m}=[-1,1]^{m}$ in $M$, up to ambient isotopy. A manifold $M$ is orientable if there are two equivalence classes, and, hence, $h$ and $h \circ r$ are not ambient isotopic. An orientation on an orientable $m$-manifold $M$ is the choice of one equivalence class.

Lemma 2.1. Let $\mathcal{P}$ be the combinatorial type of a d-polytope. Then:

(a) A complete flag on $\mathcal{P}$ induces an orientation on $|\mathcal{P}|$.

(b) If two complete flags $\mathcal{B}, \mathcal{B}^{\prime}$ differ by an elementary step, then they induce different orientations on $|\mathcal{P}|$.

(c) Two complete flags $\mathcal{B}, \mathcal{B}^{\prime}$ induce the same orientation on $|\mathcal{P}|$ if and only if they can be joined by a chain of even length.

Proof. (a) We regard $I^{d-1}$ as a polytope and, from now on, we fix a complete flag $\emptyset \prec I^{0} \prec \cdots \prec I^{d-2}$ on $I^{d-1}$. Given a complete flag $\mathcal{B}: \emptyset \prec \mathcal{F}_{0} \subset \cdots \prec \mathcal{F}_{d-1}$ on $\mathcal{P}$, 
we consider an affine embedding $\varphi: I^{d-1} \rightarrow|\mathcal{P}|$ such that $\varphi\left(I^{i}\right) \subset\left|\mathcal{F}_{i}\right|$ for all $i$. Such an embedding always exists and two such embeddings are easily shown to be ambient isotopic. So they induce the same orientation on $|\mathcal{P}|$. We call $\varphi$ an associated embedding to $\mathcal{B}$.

(b) Let $\mathcal{B}: \cdots \prec \mathcal{F}_{s} \prec \cdots$ and $\mathcal{B}^{\prime}: \cdots \prec \mathcal{F}_{s}^{\prime} \prec \cdots$ be two complete flags on $\mathcal{P}$ which differ by an elementary step, say $\mathcal{F}_{s} \neq \mathcal{F}_{s}^{\prime}$. For the case $s<d-1$, let $J^{s}$ be the $s$-face of $I^{d-1}$ different from $I^{s}$, containing $I^{s-1}$ and contained in $I^{s+1}$, and let $r$ be the (unique) reflexion of $I^{d-1}$ fixing $I^{s-1}$ pointwise and mapping $I^{s}$ to $J^{s}$. Now consider $\varphi$ to be an associated embedding to $\mathcal{B}$ such that $\varphi\left(J^{s}\right) \subset\left|\mathcal{F}_{s}^{\prime}\right|$. Then $\varphi^{\prime}=\varphi \circ r$ is an associated embedding to $\mathcal{B}^{\prime}$ and therefore $\varphi$ and $\varphi^{\prime}$ induce different orientations on $|\mathcal{P}|$. If $s=d-1$, we take $r^{\prime}$ to be the reflexion of $I^{d-1}$ such that $I^{d-2}$ and $r^{\prime}\left(I^{d-2}\right)$ are disjoint. If $\varphi$ is an associated embedding to $\mathcal{B}$, then it can be easily seen that $\varphi \circ r^{\prime}$ is ambient isotopic to an associated embedding to $\mathcal{B}^{\prime}$. Hence again $\mathcal{B}$ and $\mathcal{B}^{\prime}$ induce different orientation.

Part (c) follows inmediately from (a) and (b).

Using the previous lemma we can give the following definition.

Definition. Two oriented cycles on the combinatorial type, $\mathcal{P}$, of a polytope have the same orientation if the complete flags that they determine induce the same orientation on $|\mathcal{P}|$.

Concepts as flags, cycles, same orientation,..., defined for combinatorial types of polytopes can also be defined for affine polytopes and for polyhedral cones in the obvious way. We distinguish each case by using the appropriate notation.

The following lemmas and proposition establish relations among oriented cycles of polyhedral cones and the orientation of certain bases of the vector space $\mathbf{R}^{d+1}$. For the remainder of this section we consider $\hat{P}=\bigcap_{i=1}^{n} \hat{H}_{i}^{-}$to be a polyhedral cone of maximal dimension, $d$, in a geometric space $\left(\mathbf{R}^{d+1}, f\right)$. We suppose that no $\hat{H}_{i}$ is lightlike, and denote by $e_{i}$ the outward normal vector to $\hat{H}_{i}^{-}$. Finally we write $\hat{C}_{i}=\hat{H}_{i} \cap \hat{P}$. If $\hat{C}_{i_{1}}, \ldots, \hat{C}_{i_{d}}, \hat{C}_{i_{d+1}}$ is a maximal oriented cycle on $\hat{P}$, then for all $j$ it holds that $\operatorname{dim}\left(\hat{H}_{i_{1}} \cap \cdots \cap \hat{H}_{i_{j+1}}\right)=\operatorname{dim}\left(\hat{H}_{i_{1}} \cap \cdots \cap \hat{H}_{i_{j}}\right)-1$. Therefore, the vectors $e_{i_{1}}, \ldots, e_{i_{d+1}}$ are linearly independent, so that they form a basis of $\mathbf{R}^{d+1}$. We refer to this as the basis corresponding to the maximal cycle $\hat{C}_{i_{1}}, \ldots, \hat{C}_{i_{d}}, \hat{C}_{i_{d+1}}$.

Lemma 2.2. Let $\hat{B}$ be a complete flag on $\hat{P}$ and let $\hat{C}_{i_{1}}, \ldots, \hat{C}_{i_{d}}, \hat{C}_{i_{d+1}}$ and $\hat{C}_{j_{1}}, \ldots, \hat{C}_{j_{d}}$, $\hat{C}_{j_{d+1}}$ be maximal cycles incident to $\hat{B}$ (therefore, by definition, these cycles have the same orientation). Then the bases corresponding to these cycles have the same orientation.

Proof. First we observe that, for all $k=1, \ldots, d+1$, the linear span $L\left(e_{i_{1}}, \ldots, e_{i_{k}}\right)$ of $\left\{e_{i_{1}}, \ldots, e_{i_{k}}\right\}$ is equal to $L\left(e_{j_{1}}, \ldots, e_{j_{k}}\right)$. In fact, both subspaces are equal to the subspace $L\left(\hat{F}_{d-k}\right)^{\perp}$, where $\hat{F}_{d-k}$ is the face of dimension $d-k$ of the flag $\hat{B}$.

We denote by $E_{k}$ the subspace $L\left(e_{i_{1}}, \ldots, e_{i_{k}}\right)=L\left(\hat{F}_{d-k}\right)^{\perp}$. Then $E_{1} \subset \cdots \subset$ $E_{d+1}=\mathbf{R}^{d+1}$ and $E_{k}$ is a hyperplane of $E_{k+1}$ for each $k=1, \ldots, d$. We prove that $e_{i_{k+1}}$ and $e_{j_{k+1}}$ are in the same halfspace of $E_{k+1}$ determined by $E_{k}$. For this purpose we 
choose a basis $v_{0}, \ldots, v_{d}$ of $\hat{P}$ associated to the complete flag $\hat{B}$, that is, $v_{h} \in \hat{F}_{h} \backslash \hat{F}_{h-1}$ for all $h=0, \ldots, d-1$, and $v_{d} \notin \hat{F}_{d-1}$. These vectors are linearly independent, so that $L\left(v_{0}, \ldots, v_{k}\right)=L\left(\hat{F}_{k}\right)$. In particular, we can write

$$
E_{k}=\left\{w \in E_{k+1} \mid f\left(w, v_{d-k}\right)=0\right\} .
$$

Now $e_{i_{k+1}} \in E_{k+1} \backslash E_{k}$ and $f\left(e_{i_{k+1}}, v_{d-k}\right)<0$, because $v_{d-k}$ is a vertex of the polyhedral cone $\hat{P}$ and for the same reason $f\left(e_{j_{k+1}}, v_{d-k}\right)<0$. Hence, $e_{i_{k+1}}$ and $e_{j_{k+1}}$ are in the same halfspace of $E_{k+1}$ determined by $E_{k}$.

Summing up we have $e_{i_{1}}=e_{j_{1}}$, because $\hat{C}_{i_{1}}=\hat{C}_{j_{1}}=\hat{F}_{d-1}$; also $e_{i_{2}}=e_{j_{2}}$, because $\hat{C}_{i_{2}}=\hat{C}_{j_{2}}$. We assume, by induction, that $\left\{e_{i_{1}}, \ldots, e_{i_{k}}\right\}$ and $\left\{e_{j_{1}}, \ldots, e_{j_{k}}\right\}$ have the same orientation. Then, since $e_{i_{k+1}}$ and $e_{j_{k+1}}$ are in the same halfspace of $E_{k+1}$ determined by $E_{k}$, it is easily seen that the bases $\left\{e_{i_{1}}, \ldots, e_{i_{k+1}}\right\}$ and $\left\{e_{j_{1}}, \ldots, e_{j_{k+1}}\right\}$ have the same orientation.

Lemma 2.3. Let $\hat{B}$ and $\hat{B}^{\prime}$ be complete flags on $\hat{P}$ which differ by an elementary step. Then there exist maximal oriented cycles $\hat{C}_{1}, \ldots, \hat{C}_{d+1}$ and $\hat{C}_{1}^{\prime}, \ldots, \hat{C}_{d+1}^{\prime}$ respectively incident to $\hat{B}$ and $\hat{B}^{\prime}$ (therefore these cycles have different orientation) such that the corresponding bases of $\mathbf{R}^{d+1},\left\{e_{1}, \ldots, e_{d+1}\right\}$ and $\left\{e_{1}^{\prime}, \ldots, e_{d+1}^{\prime}\right\}$, have different orientation.

Proof. We write $\hat{B}: \emptyset \prec \hat{F}_{0} \prec \cdots \prec \hat{F}_{d-1}$ and $\hat{B}^{\prime}: \emptyset \prec \hat{F}_{0}^{\prime} \prec \cdots \prec \hat{F}_{d-1}^{\prime}$ and suppose that $\hat{F}_{r} \neq \hat{F}_{r}^{\prime}$ (consequently the rest of the faces of $\hat{B}^{\prime}$ are equal to the faces of the same dimension of $\hat{B}$ ). We choose a maximal oriented cycle $\hat{C}_{1}, \ldots, \hat{C}_{d+1}$ incident to the complete flag $\hat{B}$ and with the additional condition that $\hat{C}_{d-r+1}$ contains the face $\hat{F}_{r}^{\prime}$ (this is possible because $\hat{F}_{r}^{\prime}$ contains the face $\hat{F}_{r-1}$ ).

We now take $\hat{C}_{d-r}^{\prime}=\hat{C}_{d-r+1}, \hat{C}_{d-r+1}^{\prime}=\hat{C}_{d-r}$, and $\hat{C}_{i}^{\prime}=\hat{C}_{i}$ for all $i \neq d-r, d-r+1$. Then $\hat{C}_{1}^{\prime}, \ldots, \hat{C}_{d+1}^{\prime}$ is a maximal oriented cycle incident to $\hat{B}^{\prime}$.

These two maximal cycles differ only in the transposition of two facets; so the corresponding bases have different orientation.

Combining the previous two lemmas and using the fact that two complete flags with the same orientation can be joined by a chain of even length (Lemma 2.1(c)), we immediately obtain the following proposition.

Proposition 2.4. Let $\hat{C}_{i_{1}}, \ldots, \hat{C}_{i_{d}}, \hat{C}_{i_{d+1}}$ and $\hat{C}_{j_{1}}, \ldots, \hat{C}_{j_{d}}, \hat{C}_{j_{d+1}}$ be two maximal cycles with the same orientation. Then the corresponding bases, $\left\{e_{i_{1}}, \ldots, e_{i_{d}}, e_{i_{d+1}}\right\}$ and $\left\{e_{j_{1}}, \ldots, e_{j_{d}}, e_{j_{d+1}}\right\}$, have the same orientation.

\section{Combinatorial Type of Polyhedral Cones}

In this section we prove Proposition 3.3, which gives a sufficient condition for two sets of vectors to be, respectively, the outward normal vectors to the facets and the vertices of a polyhedral cone of a given combinatorial type. We first need some lemmas. 
Lemma 3.1. Let $K$ and $K^{\prime}$ be cell complexes and let $\varphi: K \rightarrow K^{\prime}$ be a map satisfying

(a) $\varphi$ is injective,

(b) $\varphi$ preserves cell dimensions,

(c) $C_{s} \prec C_{m}$ if and only if $\varphi\left(C_{s}\right) \prec \varphi\left(C_{m}\right)$.

Then $\varphi(K)$ is a subcomplex of $K^{\prime}$.

Proof. Let $C_{m}^{\prime}$ be an $m$-cell of $K^{\prime}$ such that $C_{m}^{\prime} \in \varphi(K)$, and let $C_{s}^{\prime} \prec C_{m}^{\prime}$; we have to show that $C_{s}^{\prime} \in \varphi(K)$. We proceed by induction on dimension $m$ of the cell.

For $m=1$, consider a 1-cell $C_{1}^{\prime}$ of $K^{\prime}$ with $C_{1}^{\prime} \in \operatorname{Im} \varphi$; let $C_{1}$ be the (unique, since $\varphi$ is injective) cell of $K$ verifying that $\varphi\left(C_{1}\right)=C_{1}^{\prime}$. As $\varphi$ preserves cell dimensions, $C_{1}$ is also a 1-cell; let $V_{1}, V_{2}$ be the two vertices of $C_{1}$. Then $\varphi\left(V_{1}\right), \varphi\left(V_{2}\right)$ are different vertices of $C_{1}^{\prime}$ by (a), (b). Then all the faces of $C_{1}^{\prime}$ belong to $\operatorname{Im} \varphi$.

We assume that the statement holds for all $k$-faces with $k<m$, that is, for each $C_{k} \in K$, all the faces of $\varphi\left(C_{k}\right)$ belong to $\varphi(K)$. Consider an $m$-cell $C_{m}^{\prime}$ of $K^{\prime}$ with $C_{m}^{\prime} \in \operatorname{Im} \varphi$. It is enough to see that all the facets of $C_{m}^{\prime}$ are contained in $\varphi(K)$, for if $C_{s}^{\prime} \prec C_{m}^{\prime}$ and $s<m-1$, then there exists an $(m-1)$-cell $C_{m-1}^{\prime}$ such that $C_{s}^{\prime} \prec C_{m-1}^{\prime} \prec C_{m}^{\prime}$, and we apply the induction hypothesis to $C_{m-1}^{\prime}$.

So let $C_{m-1}^{\prime}$ be a facet of $C_{m}^{\prime}$. Let $C_{m}$ be the $m$-cell of $K$ with $\varphi\left(C_{m}\right)=C_{m}^{\prime}$ and consider an arbitrary facet $C_{m-1,0}$ of $C_{m}$. Then the face $\varphi\left(C_{m-1,0}\right)$, denoted by $C_{m-1,0}^{\prime}$, is a facet of $C_{m}^{\prime}$ (by (b) and (c)). We consider a chain of facets in $C_{m}^{\prime}$,

$$
C_{m-1,0}^{\prime}, C_{m-1,1}^{\prime}, \ldots, C_{m-1, k}^{\prime}=C_{m-1}^{\prime},
$$

and set $C_{m-2, i}^{\prime}=C_{m-1, i}^{\prime} \cap C_{m-1, i+1}^{\prime}$.

We will prove that if $C_{m-1, i}^{\prime} \in \operatorname{Im} \varphi$, then $C_{m-2, i}^{\prime} \in \operatorname{Im} \varphi$ and $C_{m-1, i+1}^{\prime} \in \operatorname{Im} \varphi$; since $C_{m-1,0}^{\prime} \in \operatorname{Im} \varphi$, we will finally have that $C_{m-1}^{\prime} \in \operatorname{Im} \varphi$.

So suppose $C_{m-1, i}^{\prime} \in \operatorname{Im} \varphi$. First, since $C_{m-2, i}^{\prime} \prec C_{m-1, i}^{\prime}$, it follows by induction that $C_{m-2, i}^{\prime} \in \operatorname{Im} \varphi$.

Let $C_{m-2, i}$ be the $(m-2)$-cell such that $\varphi\left(C_{m-2, i}\right)=C_{m-2, i}^{\prime}$. By (c), $C_{m-2, i} \prec C_{m-1, i}$. Let $C_{m-1, i+1}$ be the unique facet of $C_{m}$ containing $C_{m-2, i}$ and different from $C_{m-1, i}$. The image of $C_{m-1, i+1}$ is an $(m-1)$-cell of $C_{m}^{\prime}$, different from $\varphi\left(C_{m-1, i}\right)=C_{m-1, i}^{\prime}$, and contains $\varphi\left(C_{m-2, i}\right)=C_{m-2, i}^{\prime}$. Then we conclude that $\varphi\left(C_{m-1, i+1}\right)=C_{m-1, i+1}^{\prime}$, and so $C_{m-1, i+1}^{\prime} \in \operatorname{Im} \varphi$.

Lemma 3.2. Let $\mathcal{P}$ and $\mathcal{P}^{\prime}$ be the combinatorial types of two d-polytopes and let $\varphi: \mathcal{P} \rightarrow \mathcal{P}^{\prime}$ be a map satisfying (a), (b), and (c) of Lemma 3.1. Suppose that $\mathcal{P}$ and $\mathcal{P}^{\prime}$ have the same number of facets. Then $\varphi$ is bijective (and hence an isomorphism).

Proof. By Lemma 3.1, $\varphi(\mathcal{P})$ is a subcomplex of $\mathcal{P}^{\prime}$. Since $\mathcal{P}$ and $\mathcal{P}^{\prime}$ have the same number of facets, all the facets of $\mathcal{P}^{\prime}$ belong to $\varphi(\mathcal{P})$. Finally, since any face of $\mathcal{P}^{\prime}$ is contained in some facet (Lemma $1.1(\mathrm{C} 1)$ ), we have $\mathcal{P}^{\prime}=\varphi(\mathcal{P})$.

Definition. (a) Let $\mathcal{P}$ be the combinatorial type of a polytope with $n$ numbered facets $\mathcal{C}_{1}, \ldots, \mathcal{C}_{n}$ and $r$ vertices $\mathcal{V}_{1}, \ldots, \mathcal{V}_{r}$. The incidence matrix of $\mathcal{P}$ is the $n \times r$ matrix 
$\mathcal{L}(\mathcal{P})=\left(l_{i j}\right)$ defined by

$$
l_{i j}= \begin{cases}0 & \text { if } \mathcal{V}_{j} \text { is a vertex of the facet } \mathcal{C}_{i}, \\ 1 & \text { otherwise. }\end{cases}
$$

(b) We say that a real matrix $H=\left(h_{i j}\right)$ has the same combinatorial type as $\mathcal{L}(\mathcal{P})$ if it verifies that $h_{i j}=0$ if and only if $l_{i j}=0$.

The incidence matrix determines the combinatorial type of the polytope up to isomorphism, since it is completely determined by its facets, its vertices, and the incident relations among them $[\mathrm{Gr}]$.

Proposition 3.3. Let $\mathcal{P}$ be the combinatorial type of a d-polytope with $n$ facets $\mathcal{C}_{1}, \ldots$, $\mathcal{C}_{n}$, and $r$ vertices, $\mathcal{V}_{1}, \ldots, \mathcal{V}_{r}$, and let $\mathcal{L}(\mathcal{P})$ be its incidence matrix. Let $\left(\mathbf{R}^{d+1}, f\right)$ be a geometric space and take $e_{1}, \ldots, e_{n} \in \mathbf{R}^{d+1}$ and $v_{1}, \ldots, v_{r} \in \mathbf{R}^{d+1}$ such that

(a) for any oriented cycle $\mathcal{C}_{i_{1}}, \ldots, \mathcal{C}_{i_{d}}$ on $\mathcal{P}$ the rank of $\left\{e_{i_{1}}, \ldots, e_{i_{d}}\right\}$ is equal to $d$;

(b) the matrix $L_{1}=\left(f\left(e_{i}, v_{j}\right)\right)_{i, j}$ has the same combinatorial type as $\mathcal{L}(\mathcal{P})$;

(c) $L_{1}$ is totally nonpositive (i.e., all non-zero entries of $L_{1}$ are negative).

Denote by $\hat{H}_{i}^{-}$the outward orthogonal halfspace to $e_{i}$, and by $\hat{P}$ the intersection $\hat{H}_{1}^{-} \cap$ $\cdots \cap \hat{H}_{n}^{-}$. Then $\hat{P}$ is a polyhedral cone that has the same combinatorial type as $\mathcal{P}$. Moreover, $v_{1}, \ldots, v_{r}$ are precisely the vertices of $\hat{P}$, and $\hat{H}_{i} \cap \hat{P}(i=1, \ldots, n)$ its facets.

Proof. The proof is divided into several steps.

(I) First, we prove that $\operatorname{rk}\left\{e_{1}, \ldots, e_{n}\right\}=d+1$ and therefore $\hat{P}$ is a polyhedral cone. Indeed, we take $\mathcal{V}_{i}$ to be an arbitrary vertex of $\mathcal{P}$ and let $\mathcal{C}_{i_{1}}, \ldots, \mathcal{C}_{i_{d}}, \mathcal{C}_{j}$ be a maximal oriented cycle incident to $\mathcal{V}_{i}$. By hypothesis (a), we have that $\operatorname{rk}\left\{e_{i_{1}}, \ldots, e_{i_{d}}\right\}=d$, that is, $\operatorname{dim}\left(\hat{H}_{i_{1}} \cap \cdots \cap \hat{H}_{i_{d}}\right)=1$. By hypothesis (b), $f\left(e_{i_{k}}, v_{i}\right)=0$ for all $k=1, \ldots, d$, and $f\left(e_{j}, v_{i}\right) \neq 0$. This implies that $v_{i} \in \hat{H}_{i_{1}} \cap \cdots \cap \hat{H}_{i_{d}}$ and $v_{i} \notin \hat{H}_{j}$. So $\hat{H}_{j} \cap \hat{H}_{i_{1}} \cap \cdots \cap \hat{H}_{i_{d}}=$ $\{0\}$ and hence $\operatorname{rk}\left\{e_{i_{1}}, \ldots, e_{i_{d}}, e_{j}\right\}=d+1$, which implies $\operatorname{rk}\left\{e_{1}, \ldots, e_{n}\right\}=d+1$.

(II) All the vectors $v_{1}, \ldots, v_{r}$ are vertices of $\hat{P}$. To see this, we consider an oriented cycle $\mathcal{C}_{i_{1}}, \ldots, \mathcal{C}_{i_{d}}$ of $\mathcal{P}$ incident to $\mathcal{V}_{i}$. We have just seen that $\hat{H}_{i_{1}} \cap \cdots \cap \hat{H}_{i_{d}}=L\left(v_{i}\right)$; on the other hand, by (c), $f\left(v_{i}, e_{j}\right) \leq 0$ for all $j=1, \ldots, n$. These conditions imply that the vector $v_{i}$ is a vertex of $\hat{P}$.

(III) Let $\mathcal{V}_{j_{0}}, \ldots, \mathcal{V}_{j_{d}}$ be a basis of vertices of $\mathcal{P}$ associated to the complete flag $\mathcal{B}: \emptyset \prec \mathcal{F}_{0} \prec \cdots \prec \mathcal{F}_{d-1}$. Then the corresponding vertices $v_{j_{0}}, \ldots, v_{j_{d}}$ are linearly independent. To prove this, we take an oriented cycle $\mathcal{C}_{i_{1}} \cdots \mathcal{C}_{i_{d}}$ of $\mathcal{P}$ incident to $\mathcal{B}$. By hypothesis (a), $\operatorname{rk}\left\{e_{i_{1}}, \ldots, e_{i_{d}}\right\}=d$, and if we call

$$
\hat{G}_{d-k}=\hat{H}_{i_{1}} \cap \cdots \cap \hat{H}_{i_{k}}, \quad \hat{G}_{d}=\mathbf{R}^{d+1},
$$

we have the sequence of linear subspaces $\hat{G}_{0} \subset \cdots \subset \hat{G}_{d-1} \subset \hat{G}_{d}$, with $\operatorname{dim} \hat{G}_{d-k}=$ $d-k+1$. Since the relation between an oriented cycle and its corresponding basis of vertices is

$$
\mathcal{V}_{j_{k}} \in \mathcal{F}_{k} \backslash \mathcal{F}_{k-1}=\left(\mathcal{C}_{i_{1}} \cap \cdots \cap \mathcal{C}_{i_{d-k}}\right) \backslash\left(\mathcal{C}_{i_{1}} \cap \cdots \cap \mathcal{C}_{i_{d-k+1}}\right),
$$


hypothesis (b) then implies that $v_{j_{k}} \in \hat{G}_{k} \backslash \hat{G}_{k-1}$, for all $k=0, \ldots, d$. Hence we conclude that $v_{j_{0}}, \ldots, v_{j_{d}}$ are linearly independent.

(IV) Let $\mathcal{F}_{r}$ be an $r$-face of $\mathcal{P}$ and let $\mathcal{C}_{i_{1}}, \ldots, \mathcal{C}_{i_{d-r}}$ be a truncated oriented cycle incident to it. We claim that

$$
\hat{F}_{r}=\hat{H}_{i_{1}} \cap \cdots \cap \hat{H}_{i_{d-r}} \cap \hat{P}
$$

is an $r$-face of $\hat{P}$; in particular, $\hat{H}_{i} \cap \hat{P}$ will be a facet of $\hat{P}$. To see this, we enlarge the cycle $\mathcal{C}_{i_{1}}, \ldots, \mathcal{C}_{i_{d-r}}$ to an oriented cycle incident to some complete flag (hence, this flag contains the face $\mathcal{F}_{r}$ ); let $\mathcal{V}_{0}, \ldots, \mathcal{V}_{d}$ be a basis associated to this flag. We have $\operatorname{dim}\left(\hat{H}_{i_{1}} \cap \cdots \cap \hat{H}_{i_{d-r}}\right)=r+1$ (by (I)), $v_{0}, \ldots, v_{r} \in \hat{F}_{r}$ (by hypothesis (b)), and these vectors are linearly independent (by (III)). This is enough to prove that $\hat{F}_{r}$ is an $r$-face.

The polyhedral cone $\hat{P}$ has the combinatorial type $\mathcal{P}^{\prime}$ of a certain $d$-polytope. Using (IV), we define the map $\varphi(\mathcal{F})=\hat{F}$, which verifies:

(i) $\varphi$ preserves face dimensions (by (IV)).

(ii) $\mathcal{F}_{r} \prec \mathcal{F}_{s}$ if and only if $\varphi\left(\mathcal{F}_{r}\right) \prec \varphi\left(\mathcal{F}_{s}\right)$. Indeed, take a complete flag containing $\mathcal{F}_{r}$ and $\mathcal{F}_{s}$ and let $\mathcal{C}_{i_{1}}, \ldots, \mathcal{C}_{i_{d+1}}$ be a maximal oriented cycle incident to this flag. Then, by (IV), $\varphi\left(\mathcal{F}_{r}\right)=\hat{H}_{i_{1}} \cap \cdots \cap \hat{H}_{i_{d-r}} \cap \hat{P}$ and $\varphi\left(\mathcal{F}_{s}\right)=\hat{H}_{i_{1}} \cap \cdots \cap \hat{H}_{i_{d-s}} \cap \hat{P}$. So $\varphi\left(\mathcal{F}_{r}\right) \prec \varphi\left(\mathcal{F}_{s}\right)$ if and only if $r \leq s$, that is, $\mathcal{F}_{r} \prec \mathcal{F}_{s}$.

(iii) $\varphi$ is injective (inmediate using (ii) above).

Also, by (IV), $\mathcal{P}^{\prime}$ has $n$ facets, which is the same number of facets as $\mathcal{P}$. By Lemma 3.2, we conclude that $\varphi$ is an isomorphism between $\mathcal{P}$ and $\mathcal{P}^{\prime}$.

\section{Main Results}

General Hypotheses. For the statements in this section we assume that $\mathcal{P}$ is the combinatorial type of a $d$-polytope with $n$ ordered facets $\mathcal{C}_{1}, \ldots, \mathcal{C}_{n} ; G$ is a real symmetric matrix of order $n$ and rank $d+1$; and $\left(\mathbf{R}^{d+1}, f\right)$ is a geometric space.

We say that a polyhedral cone $\hat{P} \subset\left(\mathbf{R}^{d+1}, f\right)$ realizes $\mathcal{P}$ and $G$ if it has the same combinatorial type as $\mathcal{P}$ and its Gram matrix is equal to $G$. We assume that the canonical basis of $\mathbf{R}^{d+1}$ is orthonormal for $f$, and coordinates of vectors are taken with respect to this basis. We denote by $F$ the matrix of the inner products of this basis and by $\operatorname{det} f$ the determinant of $F$.

Notation. Given a matrix $G$, we denote by $G\left[\begin{array}{l}i_{1} \cdots i_{r} \\ j_{1} \cdots j_{s}\end{array}\right]$ the submatrix of $G$ obtained by taking the rows $i_{1} \cdots i_{r}$ and the columns $j_{1} \cdots j_{s}$; for square submatrices we denote by $G\left(\begin{array}{l}i_{1} \cdots i_{r} \\ j_{1} \cdots j_{s}\end{array}\right)$ the determinant of that submatrix. For brevity, we write $G_{i_{1}, \cdots, i_{r}}=G\left(\begin{array}{l}i_{1} \cdots i_{r} \\ i_{1} \ldots i_{r}\end{array}\right)$.

Theorem 4.1. Suppose that the signature of $G$ equals the signature of $f$ and that $g_{i i}= \pm 1$ for all $i=1, \ldots, n$. Then there exists a polyhedral cone $\hat{P} \subset\left(\mathbf{R}^{d+1}, f\right)$ which realizes $\mathcal{P}$ and $G$ if and only if the following conditions hold:

$(R)$ (Rank) Given an arbitrary vertex of $\mathcal{P}$ and all the facets $\mathcal{C}_{i_{1}}, \ldots, \mathcal{C}_{i_{m}}$ incident to it, the submatrix $G\left[\begin{array}{l}i_{1} \cdots i_{m} \\ i_{1} \cdots i_{m}\end{array}\right]$ has rank less than or equal to $d$. 
$\left(P_{d+1}\right)$ (Principal minors of orderd +1$)$ If $\mathcal{C}_{i_{1}}, \ldots, \mathcal{C}_{i_{d+1}}$ is a maximal oriented cycle of $\mathcal{P}$, then $G_{i_{1} \cdots i_{d+1}} \operatorname{det} f>0$.

$\left(M_{d+1}\right)$ (Mixed minors of order $\left.d+1\right)$ If $\mathcal{C}_{i_{1}}, \ldots, \mathcal{C}_{i_{d}}, \mathcal{C}_{i_{d+1}}$ and $\mathcal{C}_{j_{1}}, \ldots, \mathcal{C}_{j_{d}}, \mathcal{C}_{j_{d+1}}$ are two maximal oriented cycles with the same orientation, then $G\left(\begin{array}{c}i_{1} \cdots i_{d} i_{d+1} \\ j_{1} \cdots j_{d} j_{d+1}\end{array}\right)$ det $f$ $>0$.

Moreover, if these conditions hold, the polyhedral cone $\hat{P}$ is unique up to an orthogonal transformation of $\left(\mathbf{R}^{d+1}, f\right)$.

Proof. We first prove the "only if" part. So suppose $\hat{P}=\bigcap_{i=1}^{n} \hat{H}_{i}^{-} \subset\left(\mathbf{R}^{d+1}, f\right)$ is a polyhedral cone realizing $\mathcal{P}$ and $G$. We consider the outward normal vectors $e_{i}$ of the halfspaces $\hat{H}_{i}^{-}$, so that $G=G(\hat{P})=\left(f\left(e_{i}, e_{j}\right)\right)$.

Consider $\mathcal{C}_{i_{1}}, \ldots, \mathcal{C}_{i_{m}}$ as stated in $(R)$. Since $\hat{P}$ has the same combinatorial type as $\mathcal{P}$, the vectors $e_{i_{1}}, \ldots, e_{i_{m}}$ span a linear subspace of dimension $d$ and so $\mathrm{rk} G\left[\begin{array}{l}i_{1} \cdots i_{m} \\ i_{1} \cdots i_{m}\end{array}\right] \leq d$ (the inequality being strict when the subspace spanned by $e_{i_{1}}, \ldots, e_{i_{m}}$ is lightlike). So we have proved $(R)$.

Similarly, if $\mathcal{C}_{i_{1}}, \ldots, \mathcal{C}_{i_{d+1}}$ is a maximal oriented cycle, then the vectors $e_{i_{1}}, \ldots, e_{i_{d+1}}$ are linearly independent and so the matrix $G\left[\begin{array}{l}i_{1} \cdots i_{d+1} \\ i_{1} \cdots i_{d+1}\end{array}\right]$ has a signature equal to the signature of $f$. It follows that $G_{i_{1} \cdots i_{d+1}} \operatorname{det} f>0$, and we have $\left(P_{d+1}\right)$.

Finally, let us see $\left(M_{d+1}\right)$. Let $\left\{e_{i_{1}}, \ldots, e_{i_{d}}, e_{i_{d+1}}\right\}$ and $\left\{e_{j_{1}}, \ldots, e_{j_{d}}, e_{j_{d+1}}\right\}$ be the bases corresponding to two maximal cycles with the same orientation. Denote by $E\left[i_{1} \cdots i_{r}\right]$ the matrix whose columns are the coordinates of the vectors $e_{i_{1}}, \ldots, e_{i_{r}}$. We have

$$
\begin{aligned}
G\left(\begin{array}{c}
i_{1} \cdots i_{d} i_{d+1} \\
j_{1} \cdots j_{d} j_{d+1}
\end{array}\right) \operatorname{det} f & =\operatorname{det}\left(E\left[i_{1} \cdots i_{d} i_{d+1}\right]^{t} F E\left[j_{1} \cdots j_{d} j_{d+1}\right]\right) \operatorname{det} f \\
& =(\operatorname{det} f)^{2} \operatorname{det} E\left[i_{1} \cdots i_{d} i_{d+1}\right] \operatorname{det} E\left[j_{1} \cdots j_{d} j_{d+1}\right] .
\end{aligned}
$$

Hence, $\operatorname{det} G\left(\begin{array}{l}i_{1} \cdots i_{d} i_{d+1} \\ j_{1} \cdots j_{d} j_{d+1}\end{array}\right)$ det $f>0$ if and only if the two previous bases have the same orientation and this is true by Proposition 2.4.

For the "if" part, by the hypotheses on $G$, there exists a $(d+1) \times n$ matrix $E$ such that $E^{t} F E=G$. We write $e_{1}, \ldots, e_{n}$ for the column vectors of $E$ and consider the exterior halfspaces $\hat{H}_{i}^{-}$to these vectors. We will prove that $\hat{P}=\bigcap_{i=1}^{n} \hat{H}_{i}^{-}$is a polyhedral cone that realizes $\mathcal{P}$ and $G$. By construction, the Gram matrix of $\hat{P}$ is equal to $G$.

First, since $\operatorname{rk}\left(E^{t} F E\right)=d+1$, then $\operatorname{rk} E \geq d+1$ and so rk $E=d+1$. Hence there are $d+1$ linearly independent vectors among $e_{1}, \ldots, e_{n}$ and this implies nondegeneracy, thus $\hat{P}$ is a polyhedral cone.

We use Proposition 3.3 to see that $\hat{P}$ has the same combinatorial type as $\mathcal{P}$. Then we must check that the hypotheses of that proposition hold.

First, for hypothesis (a), let $\mathcal{C}_{i_{1}}, \ldots, \mathcal{C}_{i_{d}}$ be an oriented cycle of $\mathcal{P}$ incident to a vertex $\mathcal{V}$. We choose a facet $\mathcal{C}_{j}$ not containing $\mathcal{V}$, so that $\mathcal{C}_{i_{1}}, \ldots, \mathcal{C}_{i_{d}}, C_{j}$ is now a maximal oriented cycle. By hypothesis $\left(P_{d+1}\right), G_{i_{1} \cdots i_{d} j} \neq 0$ and this implies that $e_{i_{1}}, \ldots, e_{i_{d}}, e_{j}$ are linearly independent. It follows that $\operatorname{rk}\left\{e_{i_{1}}, \ldots, e_{i_{d}}\right\}=d$. Moreover, if $\mathcal{C}_{i_{1}}, \ldots, \mathcal{C}_{i_{m}}$ are all the facets incident to $\mathcal{V}$, then the set of vectors $\left\{e_{i_{1}}, \ldots, e_{i_{m}}\right\}$ also has rank $d$. Indeed, let $\mathcal{C}_{i_{k}}$ be any facet incident to $\mathcal{V}$; if $e_{i_{1}}, \ldots, e_{i_{d}}, e_{i_{k}}$ were linearly independent, its matrix of inner products would have rank $d+1$, in contradiction with hypothesis $(R)$. Hence $e_{i_{k}} \in L\left(e_{i_{1}}, \ldots, e_{i_{d}}\right)$. 
Now we take a vector in $\mathbf{R}^{d+1}$ for each vertex of $\mathcal{P}$. In order to do this, for every vertex $\mathcal{V}_{i}$ we choose an oriented cycle $\mathcal{C}_{i_{1}}, \ldots, \mathcal{C}_{i_{d}}$ incident to it and with the property that all these cycles have the same orientation. We associate to the vertex $\mathcal{V}_{i}$ the vector $v_{i}$ defined by

$$
v_{i}=\star\left(e_{i_{1}} \wedge \cdots \wedge e_{i_{d}}\right) .
$$

(Though it will not be needed in what follows, it can be shown using $\left(M_{d+1}\right)$ that the choice of a different cycle incident to $\mathcal{V}_{i}$ yields a vector in the same ray as $v_{i}$.) The vector $v_{i}$ is nonzero since $e_{i_{1}} \wedge \cdots \wedge e_{i_{d}} \neq 0$ and $\star$ is an isomorphism. Also, by the last paragraph and the properties of the Hodge star operator (see Lemma 1.2), $f\left(v_{i}, e_{i_{k}}\right)=0$ for all the facets $\mathcal{C}_{i_{k}}$ incident to $\mathcal{V}_{i}$.

We will see now that the vectors $v_{i}$ verify hypotheses (b) and (c) of Proposition 3.3. To this end, consider the matrix $L_{1}=\left(f\left(e_{h}, v_{i}\right)\right)$. Suppose first that the entry $(h, i)$ of the matrix $L(P)$ is zero. This means that $\mathcal{V}_{i} \in \mathcal{C}_{h}$ and, as seen before, it is $f\left(e_{h}, v_{i}\right)=0$. Hence the entry $(h, i)$ of the matrix $L_{1}$ is equal to zero. Conversely, suppose that the entry $(h, i)$ of the matrix $\mathcal{L}(\mathcal{P})$ is nonzero, or equivalently $\mathcal{V}_{i} \notin \mathcal{C}_{h}$. We consider an oriented cycle $\mathcal{C}_{i_{1}}, \ldots, \mathcal{C}_{i_{d}}$ incident to $\mathcal{V}_{i}$, so that $\mathcal{C}_{i_{1}}, \ldots, \mathcal{C}_{i_{d}}, \mathcal{C}_{h}$ is a maximal oriented cycle. By hypothesis $\left(P_{d+1}\right)$ we have that $G_{i_{1} \cdots i_{d}} \neq 0$, which means that $e_{i_{1}}, \ldots, e_{i_{d}}, e_{h}$ are linearly independent. As $f\left(e_{i_{k}}, v_{i}\right)=0$ for $k=1, \ldots, d$ and $v_{i} \neq 0$, it follows that $f\left(e_{h}, v_{i}\right) \neq 0$. This shows that the matrices $\mathcal{L}(\mathcal{P})$ and $L_{1}$ have the same combinatorial type.

Finally, we see that hypothesis (c) is verified. We consider two nonzero entries $L_{1}(h, i)=f\left(e_{h}, v_{i}\right)$ and $L_{1}(k, j)=f\left(e_{k}, v_{j}\right)$ of $L_{1}$, and we check that they have the same sign, or, equivalently, $f\left(e_{h}, v_{i}\right) f\left(e_{k}, v_{j}\right)>0$. Using properties of the star operator, we easily have

$$
f\left(e_{h}, v_{i}\right) f\left(e_{k}, v_{j}\right)=G\left(\begin{array}{c}
i_{1} \cdots i_{d} h \\
j_{1} \cdots j_{d} k
\end{array}\right)(\operatorname{det} f) .
$$

Since we have chosen $\mathcal{C}_{i_{1}}, \ldots, \mathcal{C}_{i_{d}}$ and $\mathcal{C}_{j_{1}}, \ldots, \mathcal{C}_{j_{d}}$ with the same orientation, then hypothesis $\left(M_{d+1}\right)$ implies the desired inequality.

Hence all nonzero entries of $L_{1}$ have the same sign. If this is negative, we are done. Otherwise, the vectors $-v_{i}$ verify Proposition 3.3.

As particular cases of the previous theorem, we obtain a characterization of Gram matrices of spherical and finite-volume hyperbolic polytopes of a given combinatorial type.

Theorem 4.2. Suppose that all the diagonal entries of $G$ are equal to one. Then there exists a polytope $P \subset \mathbf{S}^{d}$ realizing $\mathcal{P}$ and $G$ if and only if the following conditions hold:

(R) (Rank) Given an arbitrary vertex of $\mathcal{P}$ and all the facets $\mathcal{C}_{i_{1}}, \ldots, \mathcal{C}_{i_{m}}$ incident to it, the submatrix $G\left[\begin{array}{c}i_{1} \cdots i_{m} \\ i_{1} \cdots i_{m}\end{array}\right]$ has rank equal to $d$.

$\left(P_{s}\right)$ (Principal minors of order $s$ ) If $2 \leq s \leq d+1, \mathcal{F}_{d-s}$ is a $(d-s)$-face of $\mathcal{P}$, and $\mathcal{C}_{i_{1}}, \ldots, \mathcal{C}_{i_{s}}$ is a truncated oriented cycle incident to $\mathcal{F}_{d-s}$, then $G_{i_{1} \cdots i_{s}}>0$. 
$\left(M_{d+1}\right)$ (Mixed minors of orderd +1 ) If $\mathcal{C}_{i_{1}}, \ldots, \mathcal{C}_{i_{d}}, \mathcal{C}_{i_{d+1}}$ and $\mathcal{C}_{j_{1}}, \ldots, \mathcal{C}_{j_{d}}, \mathcal{C}_{j_{d+1}}$ are two maximal cycles with the same orientation, then $G\left(\begin{array}{l}i_{1} \cdots i_{d} i_{d+1} \\ j_{1} \cdots j_{d} j_{d+1}\end{array}\right)>0$.

Moreover, the polytope P is unique up to a spherical isometry.

Proof. For the "if" part we only need to observe that conditions $\left(P_{S}\right)$ imply that $G$ is positive definite. Then Theorem 4.1 applies.

For the hyperbolic case, the polyhedral cones obtained in Theorem 4.1 do not necessarily produce finite-volume hyperbolic polytopes. In fact, $P=\hat{P} \cap \mathbf{H}^{d}$ has finite volume if and only if all the vertices of $\hat{P}$ meet $\mathbf{H}^{d}$ or $\partial \mathbf{H}^{d}$. Thus, essentially, the inner product of any two of these vertices must be negative. This is the meaning of conditions $\left(P_{d}\right)$ and $\left(M_{d}\right)$ in Theorem 4.4 below. Before stating it, we give the following lemma which establishes that the vertices of a polyhedral cone can be determined (from its facets) by taking cycles with the same orientation and applying the Hodge star operator.

Lemma 4.3. Let $\hat{P} \subset\left(\mathbf{R}^{d+1}, f\right)$ be a polyhedral cone of dimension d; let $V_{1}, \ldots, V_{r} \in$ $\mathbf{R}^{d+1}$ be its vertices and let $e_{1}, \ldots, e_{n}$ be the outward normal vectors to its facets. For each vertex $V_{i}$ we choose a cycle $\hat{C}_{i_{1}}, \ldots, \hat{C}_{i_{d}}$ incident to it and such that all these cycles have the same orientation. Define $v_{i}=\star\left(e_{i_{1}} \wedge \cdots \wedge e_{i_{d}}\right)$. Then there exist positive constants $\lambda_{1}, \ldots, \lambda_{r}$ and $\varepsilon= \pm 1$ such that $\lambda_{i} V_{i}=\varepsilon v_{i}$, for all $i=1, \ldots, r$.

Proof. The vector $v_{i}=\star\left(e_{i_{1}} \wedge \cdots \wedge e_{i_{d}}\right)$ is orthogonal to the vectors $e_{i_{1}}, \ldots, e_{i_{d}}$; in order to prove that $v_{i}=\lambda_{i} V_{i}$, with $\lambda_{i}>0$, it suffices to see that $f\left(v_{i}, e_{h}\right)<0$ for all $h$ such that $\mathcal{V}_{i} \notin \mathcal{C}_{h}$.

Let $V_{j}$ be any vertex of $\hat{P}$ and let $\hat{C}_{j_{1}}, \ldots, \hat{C}_{j_{d}}$ be the cycle chosen in the statement. Consider facets $\hat{C}_{h}, \hat{C}_{k}$ respectively not incident to $V_{i}, V_{j}$. Then, using the same notation as in the proof of Theorem 4.1, we have $f\left(v_{i}, e_{h}\right)=\operatorname{det} E\left[i_{1} \cdots i_{d} h\right]$ and $f\left(v_{j}, e_{k}\right)=$ $\operatorname{det} E\left[j_{1} \cdots j_{d} k\right]$.

By Proposition 2.4, the bases $\left\{e_{i_{1}}, \ldots, e_{i_{d}}, e_{h}\right\}$ and $\left\{e_{j_{1}}, \ldots, e_{j_{d}}, e_{k}\right\}$ have the same orientation and so $f\left(v_{i}, e_{h}\right) f\left(v_{j}, e_{k}\right)>0$. Consequently, if $f\left(v_{i}, e_{h}\right)<0$, then we have $\lambda_{i} V_{i}=v_{i}$ for all $i$, and otherwise we have $\lambda_{i} V_{i}=-v_{i}$ for all $i$.

Theorem 4.4. Suppose that all the diagonal entries of $G$ are equal to one. Let $\mathcal{W}$ be a subset of the set of vertices of $\mathcal{P}$. Then there exists a finite-volume polytope $P \subset \mathbf{H}^{d}$ realizing $\mathcal{P}$ and $G$, and whose vertices on $\partial \mathbf{H}^{d}$ correspond to those in $\mathcal{W}$, if and only if the following conditions hold:

(R) (Rank) Given an arbitrary vertex of $\mathcal{P}$ and all the facets $\mathcal{C}_{i_{1}}, \ldots, \mathcal{C}_{i_{m}}$ incident to it, the submatrix $G\left[\begin{array}{l}i_{1} \cdots i_{m} \\ i_{1} \cdots i_{m}\end{array}\right]$ has rank less than or equal to $d$.

$\left(P_{s}\right)$ (Principal minors of order $s$ ) If $2 \leq s \leq d, \mathcal{F}_{d-s}$ is a $(d-s)$-face of $\mathcal{P}$, and $\mathcal{C}_{i_{1}}, \ldots, \mathcal{C}_{i_{s}}$ is a truncated oriented cycle incident to $\mathcal{F}_{d-s}$, then $G_{i_{1} \cdots i_{s}} \geq 0$ and the equality holds if and only if $s=d$ and $\mathcal{F}_{0}$ is a vertex in $\mathcal{W}$.

$\left(P_{d+1}\right)$ (Principal minors of order $\left.d+1\right)$ If $\mathcal{C}_{i_{1}}, \ldots, \mathcal{C}_{i_{d+1}}$ is a maximal oriented cycle of $\mathcal{P}$, then $G_{i_{1} \cdots i_{d+1}}<0$. 
$\left(M_{d}\right)$ (Mixed minors of order d) If $\mathcal{C}_{i_{1}}, \ldots, \mathcal{C}_{i_{d}}$ and $\mathcal{C}_{j_{1}}, \ldots, \mathcal{C}_{j_{d}}$ are two cycles of $\mathcal{P}$ with the same orientation and incident to different vertices, then $G\left(\begin{array}{c}i_{1} \cdots i_{d} \\ j_{1} \cdots j_{d}\end{array}\right)>0$.

$\left(M_{d+1}\right)$ (Mixed minors of order $d+1$ ) If $\mathcal{C}_{i_{1}}, \ldots, \mathcal{C}_{i_{d}}, \mathcal{C}_{i_{d+1}}$ and $\mathcal{C}_{j_{1}}, \ldots, \mathcal{C}_{j_{d}}, \mathcal{C}_{j_{d+1}}$ are

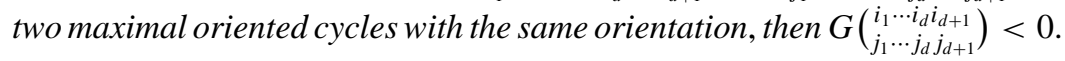

Moreover, $P$ is unique up to a hyperbolic isometry.

Proof. First, let $P=\hat{P} \cap \mathbf{H}^{d}$ be a $d$-polytope satisfying the hypotheses of the theorem. We consider the outward normal vectors $e_{i}$ to the facets $C_{i}$ of $P$. By Theorem 4.1, $(R)$, $\left(P_{d+1}\right)$, and $\left(M_{d+1}\right)$ hold.

To see $\left(P_{s}\right)$, take $\mathcal{C}_{i_{1}}, \ldots, \mathcal{C}_{i_{s}}$ as a truncated oriented cycle incident to $\mathcal{F}_{d-s}$. Since $P$ has finite volume, the face $\hat{F}_{d-s}$ of $\hat{P}$ corresponding to $\mathcal{F}_{d-s}$ either meets $\mathbf{H}^{d}$ or is a vertex on $\partial \mathbf{H}^{d}$ (in the latter case, $s=d$ and $\mathcal{F}_{0}$ belongs to $\mathcal{W}$ ). Then the quadratic form $f$ restricted to the orthogonal subspace, spanned by $e_{i_{1}}, \ldots, e_{i_{s}}$, is either positive definite or semidefinite, and so is the submatrix $G\left[\begin{array}{c}i_{1} \cdots i_{d} \\ i_{1} \cdots i_{d}\end{array}\right]$. Hence we have $\left(P_{s}\right)$.

Finally, to see $\left(M_{d}\right)$ we consider $\mathcal{C}_{i_{1}}, \ldots, \mathcal{C}_{i_{d}}$ and $\mathcal{C}_{j_{1}}, \ldots, \mathcal{C}_{j_{d}}$ to be two cycles of $\mathcal{P}$ with the same orientation and incident to different vertices $\mathcal{V}_{i}$ and $\mathcal{V}_{j}$. Let $V_{i}$ and $V_{j}$ be the corresponding vertices of $\hat{P}$. By Lemma 4.3 , there exist $\lambda_{i}>0, \lambda_{j}>0$, and $\varepsilon= \pm 1$ such that $V_{i}=\varepsilon \lambda_{i} \star\left(e_{i_{1}} \wedge \cdots \wedge e_{i_{d}}\right)$ and $V_{j}=\varepsilon \lambda_{j} \star\left(e_{j_{1}} \wedge \cdots \wedge e_{j_{d}}\right)$. Applying Lemma 1.2(c), we have

$$
\begin{aligned}
f\left(V_{i}, V_{j}\right) & =\lambda_{i} \lambda_{j} f\left(\star\left(e_{i_{1}} \wedge \cdots \wedge e_{i_{d}}\right), \star\left(e_{j_{1}} \wedge \cdots \wedge e_{j_{d}}\right)\right) \\
& =\lambda_{i} \lambda_{j}(\operatorname{det} f)\left(\bigwedge^{d} f\right)\left(e_{i_{1}} \wedge \cdots \wedge e_{i_{d}}, e_{j_{1}} \wedge \cdots \wedge e_{j_{d}}\right)=-\lambda_{i} \lambda_{j} G\left(\begin{array}{c}
\left(i_{1} \cdots i_{d}\right. \\
j_{1} \cdots j_{d}
\end{array}\right) .
\end{aligned}
$$

Since $P$ has finite volume, $f\left(V_{i}, V_{j}\right)<0$. As $\lambda_{i} \lambda_{j}>0$, we finally get that $G\left(\begin{array}{l}i_{1} \cdots i_{d} \\ j_{1} \cdots j_{d}\end{array}\right)>0$.

We now prove the "if" part. Using hypotheses $\left(P_{s}\right)$ and $\left(P_{d+1}\right)$ it is easily seen that the signature of $G$ is $(d, 1)$. Then, by Theorem 4.1, there exists a polyhedral cone $\hat{P} \subset\left(\mathbf{R}^{d+1}, f\right)$ realizing $\mathcal{P}$ and $G$. In the proof of this theorem we found the vectors $v_{1}, \ldots, v_{r}$, which were the vertices of $\hat{P}$. To finish the proof we must see that the norm of a vertex $v_{i}$ is zero if it corresponds to a vertex $\mathcal{V}_{i} \in \mathcal{W}$ and negative otherwise, and that $f\left(v_{i}, v_{j}\right)<0$ for all $i \neq j$. So let $\mathcal{C}_{i_{1}}, \ldots, \mathcal{C}_{i_{d}}$ and $\mathcal{C}_{j_{1}}, \ldots, \mathcal{C}_{j_{d}}$ be cycles with the same orientation incident to the vertices $\mathcal{V}_{i}, \mathcal{V}_{j}$. By the choice of $v_{i}, v_{j}$ and with the same calculation as in the "only if" part, we have that

$$
f\left(v_{i}, v_{j}\right)=-G\left(\begin{array}{l}
i_{1} \cdots i_{d} \\
j_{1} \cdots j_{d}
\end{array}\right)
$$

Then we have the result, using the hypotheses $\left(P_{d}\right)$ and $\left(M_{d}\right)$.

From Theorems 4.2 and 4.4 we obtain the classical result for simplices (see [Vi2] and [Mi]):

Corollary 4.5. Let $G=\left(g_{i j}\right)$ be a real symmetric matrix with order and rank equal to $d+1$ and $g_{i i}=1$ for all $i$. Then

(I) There exists a d-simplex $T \subset \mathbf{S}^{d}$ realizing $G$ if and only if $G$ is positive definite. 
(II) There exists a compact hyperbolic simplex realizing $G$ if and only if

$\left(P_{d+1}\right) \operatorname{det} G<0$

$\left(P_{d}\right)$ all proper principal minors of $G$ are positive;

$\left(M_{d}\right)$ all the entries of the matrix $G^{-1}$ are negative.

\section{Applications}

1. In order to obtain an analogous result to Theorem 4.4 for general hyperbolic polytopes (i.e., not necessarily of finite volume), it is necessary to study the relative position of each face of the polyhedral cone with the hyperbolic space.

2. In Theorem 4.1 we can replace "combinatorial type $\mathcal{P}$ of a $d$-polytope" by "linear convex cell complex $\mathcal{S}$, whose underlying space is homeomorphic to a $(d-1)$-sphere." It can be shown that such a cell complex $\mathcal{S}$ verifies Lemma 1.1. Then one can immediately check that an analogous result to Theorem 4.1 is obtained. This result provides a criterion to decide whether the complex $\mathcal{S}$ is polytopal, that is, isomorphic to the boundary of a polytope. Indeed, $\mathcal{S}$ is polytopal if and only if there exists a matrix $G$ verifying all conditions in Theorem 4.1. Cell complexes whose underlying spaces are homeomorphic to spheres are more general than combinatorial spheres (in the sense of the piecewise-linear topology, see [Hu]). Using the theory of oriented matroids, Bokowski and Sturmfels [BS] gave a practical algorithm to decide whether a combinatorial sphere is polytopal or not.

3. The results in Section 4 may also provide a new approach to solving the generalized Andreev's theorem for some particular cases of polyhedra: given the combinatorial type of a polyhedron and a number $\alpha_{i j} \in(0, \pi)$ assigned to each edge, does there exist a finite-volume hyperbolic (or spherical) polyhedron with the given combinatorial type and whose dihedral angles are the given numbers? The way to do this, following our method, would be: first get all the matrices $G=\left(g_{i j}\right)$ with $g_{i j}=-\cos \alpha_{i j}$ and with rk $G=4$; second, check whether some of these matrices verify the conditions in Theorems 4.4 or 4.2. The first part is, obviously, the most difficult. In [Di] we solved the generalized Andreev's theorem for the tetrahedron's descendants, that is, the polyhedra obtained by taking an initial tetrahedron and successively truncating vertices. Since tetrahedron's descendants are simple polyhedra (that is, there are exactly three faces incident to each vertex) it is known from Cauchy's lemmas that the dihedral angles determine the polyhedron (see $[\mathrm{RH}]$ ). Our method provides an alternative proof for this fact. For the triangular prism, the first polyhedron in this family, we described the space of realizable dihedral angles by giving the explicit polynomial equalities and inequalities.

\section{Example: the Lambert Cube}

We now illustrate the general method with a concrete example. Consider the cube and assign angles $\alpha, \beta, \gamma \in(0, \pi)$ to the three edges shown in Fig. 1 and $\pi / 2$ to the other edges. The cube marked in this way is called the Lambert cube. The Lambert cube is used, for instance, to give structure of orbifold (or, more generally, of conic manifold) to $\mathcal{P}^{3}$ with singularity on Borromeo's link [Th], [HLMW].

A finite-volume hyperbolic Lambert cube is easily seen to be compact and to have 


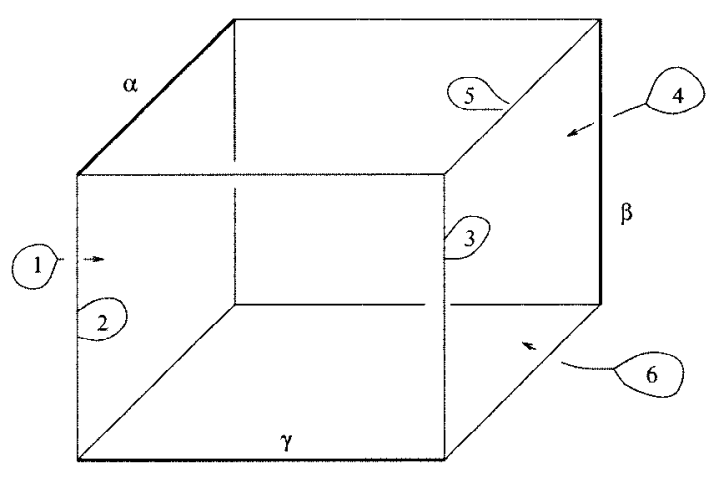

Fig. 1. The Lambert cube.

angles $\alpha, \beta, \gamma$ less than $\pi / 2$. Thus, all its dihedral angles are nonobtuse and Andreev's theorem applies.

Proposition 5.1. There exists a compact hyperbolic Lambert cube with angles $\alpha, \beta$, and $\gamma$ if and only if $\alpha, \beta, \gamma \in(0, \pi / 2)$.

Proof. Applying Andreev's theorem [An1], the angles $\pi / 2, \pi / 2, \pi / 2, \alpha$ of the quadrangular prismatic element determined by the faces $C_{1}, C_{5}, C_{3}, C_{6}$, sum to less than $2 \pi$. Thus, $\alpha<\pi / 2$. In an analogous way, $\beta, \gamma<\pi / 2$. With this, all the conditions in Andreev's theorem are verified.

We give a different proof by using the Gram matrix, which also shows that any Lambert cube is compact. This also provides a characterization of the angles of the spherical Lambert cubes.

\section{Proposition 5.2.}

(a) There exists a hyperbolic Lambert cube with angles $\alpha, \beta, \gamma$ if and only if $\alpha, \beta, \gamma \in$ $(0, \pi / 2)$.

(b) There exists a spherical Lambert cube with angles $\alpha, \beta, \gamma$ if and only if $\alpha, \beta, \gamma \in$ $(\pi / 2, \pi)$.

In the proof we use the following easy lemma.

Lemma 5.3. Let $G$ be a real symmetric matrix of order $n$. Then:

(a) $\mathrm{rk} G \leq m$ if and only if all principal minors $G_{i_{1} \cdots i_{r}}$ of order $r>m$ are equal to zero.

(b) If $\mathrm{rk} G=m$, then $\operatorname{rk}\left(\bigwedge^{r} G\right)=\left(\begin{array}{l}m \\ r\end{array}\right)$, where $\bigwedge^{r} G$ is the matrix of minors of order $r$ of $G$. 
Proof of Proposition 5.2. The Gram matrix of a hyperbolic or spherical Lambert cube is

$$
\left(\begin{array}{cccccc}
1 & 0 & u & 0 & a & 0 \\
0 & 1 & 0 & v & 0 & c \\
u & 0 & 1 & b & 0 & 0 \\
0 & v & b & 1 & 0 & 0 \\
a & 0 & 0 & 0 & 1 & w \\
0 & c & 0 & 0 & w & 1
\end{array}\right),
$$

where $a=-\cos \alpha, b=-\cos \beta, c=-\cos \gamma$. We apply Theorem 4.1, either with $f$ positive definite (for the spherical case) or when the signature of $f$ is $(3,1)$ (for the hyperbolic case).

We observe that conditions $\left(P_{4}\right)$ and $\left(M_{4}\right)$ in Theorem 4.1 can be gathered together as the entries of a certain submatrix $G_{0}$ of order 12 of the matrix $\wedge^{4} G$. Indeed, we consider the twelve different maximal cycles in the cube (we consider two cycles to be equal if one is obtained from the other by reordering its faces) and such that all of them have the same orientation. Then the entries of $G_{0}$ are defined to be the minors $G\left(\begin{array}{l}i_{1} i_{2} i_{3} i_{4} \\ j_{1} j_{2} j_{3} j_{4}\end{array}\right)$ where $\mathcal{C}_{i_{1}}, \ldots, \mathcal{C}_{i_{4}}$ and $\mathcal{C}_{j_{1}}, \ldots, \mathcal{C}_{j_{4}}$ are two of the chosen maximal oriented cycles. With these conventions, conditions $\left(P_{4}\right)$ and $\left(M_{4}\right)$ establish that the entries of $G_{0}$ are either strictly positive, for the spherical case, or strictly negative, for the hyperbolic case.

On the other hand, by Lemma $5.3(\mathrm{~b}), \operatorname{rk} G=4$ if and only if $\operatorname{rk}\left(\bigwedge^{4} G\right)=1$. Also, if $\operatorname{rk} G=4$, all nonzero principal minors of order 4 have the same sign. Thus, assuming rk $G=4$, and since $G$ is symmetric, an equivalent condition to conditions $\left(P_{4}\right)$ and $\left(M_{4}\right)$ is that one diagonal entry of $G_{0}$ and one off-diagonal entry in each row or column have all the appropiate signs. In our case, we can regard the signs of the following twelve entries of $G_{0}$ :

$$
\begin{array}{llrl}
G_{1254} & =\left(1-a^{2}\right)\left(1-v^{2}\right), & \\
G\left(\begin{array}{l}
1253 \\
1254
\end{array}\right)=b\left(1-a^{2}\right), & G\left(\begin{array}{c}
1254 \\
1256
\end{array}\right)=-v c\left(1-a^{2}\right), & G\left(\begin{array}{c}
1253 \\
2354
\end{array}\right)=-b u, \\
G\left(\begin{array}{c}
1253 \\
2356
\end{array}\right)=-a w, & G\left(\begin{array}{c}
2354 \\
3451
\end{array}\right)=b u v, & G\left(\begin{array}{c}
2356 \\
3456
\end{array}\right)=v\left(w^{2}-1\right), \\
G\left(\begin{array}{l}
1254 \\
4156
\end{array}\right)=c\left(1-a^{2}\right), & G\left(\begin{array}{c}
2356 \\
1623
\end{array}\right)=a\left(1-c^{2}\right), & G\left(\begin{array}{c}
1254 \\
1624
\end{array}\right)=w\left(v^{2}-1\right), \\
G\left(\begin{array}{c}
2356 \\
2634
\end{array}\right)=c v w, & G\left(\begin{array}{l}
1623 \\
3641
\end{array}\right)=v\left(u^{2}-1\right) . &
\end{array}
$$

First, suppose there is a polyhedral cone $\hat{P} \subset\left(\mathbf{R}^{4}, f\right)$, with the combinatorial type of a Lambert cube, and the signature of $f$ equal to $(3,1)$. Since $a, b, c \in(-1,1)$, then conditions $\left(P_{4}\right)$ and $\left(M_{4}\right)$ of Theorem 4.1 for the twelve minors above imply that $a, b, c<0, u, v, w<0$, and $u^{2}, v^{2}, w^{2}>1$. Hence $\alpha, \beta, \gamma<\pi / 2$. Moreover, we can check that $G$ verifies conditions $\left(P_{3}\right)$ and $\left(M_{3}\right)$ of Theorem 4.4, when $\mathcal{W}=\varnothing$, and therefore $\hat{P} \cap \mathbf{H}^{3}$ is compact. Similarly, if there is a spherical Lambert cube, then $a, b, c>0, u, v, w<0$, and $u^{2}, v^{2}, w^{2}<1$ and so $\alpha, \beta, \gamma>\pi / 2$.

Conversely, suppose we are given $\alpha, \beta, \gamma \in(0, \pi / 2)$, or equivalently $a, b, c \in$ $(-1,0)$. We will find values for $u, v, w$ in the interval $(-\infty,-1)$ and such that $\operatorname{rk} G=4$. Then it is easy to see that all the conditions in Theorem 4.4 are verified and hence there exists a compact hyperbolic Lambert cube with angles $\alpha, \beta, \gamma$. By Lemma 5.3(a), rk $G=4$ 
if all the principal minors of $G$ of order 5 and 6 are equal to zero (and that there is one nonzero minor of order 4 ). We compute these determinants:

$$
\begin{aligned}
G_{12345}= & u^{2} v^{2}-u^{2}-v^{2}+a^{2} v^{2}+a^{2} b^{2}-a^{2}-b^{2}+1, \\
G_{12346}= & u^{2} v^{2}-v^{2}-u^{2}+c^{2} u^{2}+c^{2} b^{2}-c^{2}-b^{2}+1, \\
G_{12356}= & u^{2} w^{2}-u^{2}-w^{2}+c^{2} u^{2}+a^{2} c^{2}-a^{2}-c^{2}+1, \\
G_{12456}= & v^{2} w^{2}-v^{2}-w^{2}+a^{2} v^{2}+a^{2} c^{2}-a^{2}-c^{2}+1, \\
G_{13456}= & u^{2} w^{2}-u^{2}-w^{2}+b^{2} w^{2}+a^{2} b^{2}-a^{2}-b^{2}+1, \\
G_{23456}= & v^{2} w^{2}-v^{2}-w^{2}+b^{2} w^{2}+b^{2} c^{2}-b^{2}-c^{2}+1, \\
\operatorname{det} G= & -u^{2} v^{2} w^{2}+u^{2} v^{2}+u^{2} w^{2}+v^{2} w^{2}-u^{2}-v^{2}-w^{2}+c^{2} u^{2}+a^{2} v^{2}+b^{2} w^{2} \\
& -2 a b c u v w-a^{2} b^{2} c^{2}+a^{2} b^{2}+a^{2} c^{2}+b^{2} c^{2}-a^{2}-b^{2}-c^{2}+1 .
\end{aligned}
$$

We can determine $u, v$ verifying $G_{12345}=0$ and $G_{12346}=0$ by computing the resultant of these two polynomials. We obtain

$$
\operatorname{res}\left(G_{12345}, G_{12346}, u\right)=\operatorname{res}\left(G_{12456} G_{23456}, w\right)=q(v)^{2},
$$

where $q(v)$ is the biquadratic polynomial

$$
q(v)=a^{2} v^{4}+\left(a^{2} b^{2}+a^{2} c^{2}-2 a^{2}-b^{2} c^{2}\right) v^{2}+a^{2}\left(1-c^{2}\right)\left(1-b^{2}\right) .
$$

Setting $A=a^{2}, B=a^{2} b^{2}+a^{2} c^{2}-2 a^{2}-b^{2} c^{2}, C=a^{2}\left(1-c^{2}\right)\left(1-b^{2}\right)=a^{2} b^{2} c^{2}+$ $a^{2}-a^{2} c^{2}-a^{2} b^{2}$, and $D=B^{2}-4 A C$, we find the four roots of $q(v)$ :

$$
\begin{array}{ll}
v_{1}=-\sqrt{\frac{-B+\sqrt{D}}{2 A}}, & v_{2}=\sqrt{\frac{-B+\sqrt{D}}{2 A}}, \\
v_{3}=-\sqrt{\frac{-B-\sqrt{D}}{2 A}}, & v_{4}=\sqrt{\frac{-B-\sqrt{D}}{2 A}} .
\end{array}
$$

First we see that the four roots are real numbers, and $v_{1}^{2}, v_{2}^{2}>1$ and $v_{3}^{2}, v_{4}^{2}<1$. This is done in the following lemma:

Lemma 5.4. We suppose $a, b, c \in(-1,0) \cup(0,1)$; then

(a) $B<0$,

(b) $D$ is symmetric in $a, b$, c and strictly positive; also $-B-\sqrt{D}>0$,

(c) $v_{1}^{2}=v_{2}^{2}>1, v_{3}^{2}=v_{4}^{2}<1$.

Proof. (a) $B=a^{2} b^{2}+a^{2} c^{2}-2 a^{2}-b^{2} c^{2}=-a^{2}\left(1-b^{2}\right)-a^{2}\left(1-c^{2}\right)-b^{2} c^{2}<0$.

(b) Expanding $D=B^{2}-4 A C$ we have

$$
\begin{aligned}
B^{2}-4 A C & =a^{4} b^{4}+a^{4} c^{4}+b^{4} c^{4}-2 a^{4} b^{2} c^{2}-2 a^{2} b^{4} c^{2}-2 a^{2} b^{2} c^{4}+4 a^{2} b^{2} c^{2} \\
& =\left(a^{2} b^{2}-a^{2} c^{2}-b^{2} c^{2}\right)^{2}+4 a^{2} b^{2} c^{2}\left(1-c^{2}\right)>0
\end{aligned}
$$


(the first expression for $B^{2}-4 A C$ shows its symmetry). On the other hand, since $B<0$, $-B>\sqrt{B^{2}-4 A C}$ is equivalent to $B^{2}>B^{2}-4 A C$, which is true since $A$ and $C$ are nonnegative.

(c) $v_{1}^{2}$ and $v_{3}^{2}$ are the two roots of the quadratic polynomial $Q(x)=A x^{2}+B x+C$, and clearly $v_{3}^{2}<v_{1}^{2}$. Since $A=a^{2}>0, y=Q(x)$ is a convex parabola, and we observe that $Q(1)=A+B+C=-b^{2} c^{2}\left(1-a^{2}\right)<0$. Thus, we deduce that $v_{3}^{2}<1<v_{1}^{2}$.

Since the matrix $G$ must verify conditions $\left(P_{4}\right)$ and $\left(M_{4}\right), v$ must be negative and less than -1 . Hence we take $v=v_{1}$.

Because of the symmetry of the Lambert cube, we obtain in an analogous way that the only possible values for $u$ and $w$ are

$$
\begin{aligned}
& u=-\sqrt{\frac{-B^{\prime}+\sqrt{D}}{2 A^{\prime}}}, \quad \text { where } \quad A^{\prime}=c^{2}, \quad B^{\prime}=c^{2} b^{2}+c^{2} a^{2}-2 c^{2}-b^{2} a^{2}, \\
& w=-\sqrt{\frac{-B^{\prime \prime}+\sqrt{D}}{2 A^{\prime \prime}}}, \quad \text { where } \quad A^{\prime \prime}=b^{2}, \quad B^{\prime \prime}=b^{2} a^{2}+b^{2} c^{2}-2 b^{2}-a^{2} c^{2} .
\end{aligned}
$$

It remains to prove that for these values of $u, v, w$, the matrix $G$ has rank 4 . It can be checked directly that all principal minors of order 5 are equal to zero. Using this, we get the following expression for the determinant of $G$ :

$$
\operatorname{det}(G)=-u^{2} v^{2} w^{2}+u^{2}+v^{2}+w^{2}-2 a b c u v w-a^{2} b^{2} c^{2}+a^{2}+b^{2}+c^{2}-2 .
$$

Substituting $u, v, w$ by their values, we obtain

$$
\operatorname{det}(G)=\frac{P+4 \sqrt{D}}{4}+2 a b c \sqrt{\frac{Q+P \sqrt{D}}{8 A A^{\prime} A^{\prime \prime}}},
$$

where $P=B B^{\prime}+B B^{\prime \prime}+B^{\prime} B^{\prime \prime}+D$ and $Q=-B B^{\prime} B^{\prime \prime}-\left(B+B^{\prime}+B^{\prime \prime}\right) D$, and so both polynomials are positive for $a, b, c \in(-1,0)$ (Lemma 5.4). By hypothesis, $a, b, c<0$, so that $\sqrt{A A^{\prime} A^{\prime \prime}}=-a b c$. Then $\operatorname{det}(G)=0$ is equivalent to

$$
P+4 \sqrt{D}=\sqrt{8} \sqrt{Q+P \sqrt{D}}
$$

Since $P$ and $Q$ are positive, the previous expression is equivalent to

$$
(P+4 \sqrt{D})^{2}=8 Q+P \sqrt{D}
$$

and this is readily seen to hold.

Finally, if we are given $\alpha, \beta, \gamma \in(\pi / 2, \pi)$, then we take $v=v_{3}, u=$ $-\sqrt{\left(-B^{\prime}-\sqrt{D}\right) / 2 A^{\prime}}$, and $w=-\sqrt{\left(-B^{\prime \prime}-\sqrt{D}\right) / 2 A^{\prime \prime}}$, and proceed in an analogous way to get a matrix $G$ which verifies the conditions of Theorem 4.2. Hence there exists a spherical Lambert cube with the given angles. 


\section{Acknowledgments}

The content of this paper forms part of the author's doctoral dissertation. I would like to thank J.M. Montesinos for proposing this problem to me and E. Arrondo and I. Rivin for many helpful discussions and comments.

\section{References}

[Al] A.D. Alexandrow, Konvexe Polyeder, Berlin: Akademie Verlag, 1958 (original in Russian: GITTL, Moscow, 1950).

[An1] E.M. Andreev, On convex polyhedra in Lobachevskii spaces, Math. USSR-Sb. 10 (1970), 413-440.

[An2] E.M. Andreev, Convex polyhedra of finite volume in Lobachebskii space, Math. USSR-Sb. 12 (1970), 255-259.

[An3] E.M. Andreev, The intersection of the plane boundaries of a polytope with acute angles, Mat. Zametki 8 (1970), 521-527.

[BL] M.M. Bayer, C.W. Lee, Combinatorial aspects of convex polytopes, in Handbook of Convex Geometry, ed. by P.M. Gruber and J.M. Wills, Amsterdam: North-Holland, 1993.

[BS] J. Bokowski, B. Sturmfels, Polytopal and nonpolytopal spheres, an algorithmic approach, Israel J. Math., 57(3) (1987), 257-271.

[Ca] A.L. Cauchy, Sur les polygones et polyèdres, J. Ecole Polytechnique 16 (1813), 87-99.

[Co] H.S.M. Coxeter, Discrete groups generated by reflections, Ann. of Math. 35(2) (1934), 588-621.

[Di] R. Díaz, Matrices de Gram y espacios de ángulos diédricos de poliedros, Ph.D. Thesis, Universidad Complutense de Madrid, June 1996.

[Gr] B. Grünbaum, Convex Polytopes, Pure and Applied Mathematics, Vol. XVI, New York: Interscience, 1967.

[HLMW] H.M. Hilden, M.T. Lozano, J.M. Montesinos, W.C. Whitten, On universal groups and threemanifolds, Invent. Math. 87 (1987), 441-456.

[Hu] J.F.P. Hudson, Piecewise Linear Topology, New York: Benjamin, 1969.

[Iv] B. Iversen, Hyperbolic Geometry, Cambridge: Cambridge University Press, 1992.

[La] F. Lannér, On complexes with transitive groups of automorphisms, Comm. Sem. Math. Univ. Lund 11 (1950), 1-71.

[Mi] J. Milnor, The Schläfli differential equality, in Collected papers, Vol 1, Houston: Publish or Perish, 1994.

[RH] I. Rivin, C.D. Hodgson, A characterization of compact convex polyhedra in hyperbolic 3-space, Invent. Math. 111 (1993), 77-111.

[Ri1] I. Rivin, On geometry of convex ideal polyhedra in hyperbolic 3-space, Topology 32(1) (1993), 87-92.

[Ri2] I. Rivin, A characterization of ideal polyhedra in hyperbolic 3-space, Ann. of Math. 143(1) (1996), 51-70.

[RS] C.P. Rourke, B.J. Sanderson, Introduction to Piecewise-Linear Topology, New York: SpringerVerlag, 1972 .

[Th] W. Thurston, The Geometry and Topology of 3-manifolds, Princeton University Notes, preprint.

[Vi1] E.B. Vinberg, Hyperbolic reflection groups, Russian Math. Surveys 40(1) (1985), 31-75.

[Vi2] E.B. Vinberg, Volumes of non-Euclidean polyhedra, Russian Math. Surveys 48(2) (1993), 15-45.

[Vi3] E.B. Vinberg (ed.), Geometry II, New York: Springer-Verlag, 1993.

Received June 20, 1997, and in revised form November 21, 1997. 